in the Massachusetts Institute of Technology; Mr. F. E. KNOWLES as instructor in mathematics in the University of Oklahoma; Mr. W. O. BEAL as instructor in mathematics in Illinois College ; Mr. O. S. SteTson as instructor in mathematics in Syracuse University ; Mr. J. W. BRIsTER as associate professor of mathematics in the University of Nashville; Mr. M. A. Mackenzie, of Trinity College, Toronto, as associate professor of mathematics in the University of Toronto.

THE following catalogues of second-hand mathematical works have recently been issued : B. Quaritch, 15 Piccadilly, London, catalogue No. 225, 114 numbers, mostly very rare works; Theodor Ackermann, Promenadenplatz 10, Munich, catalogue 175a, 2 pages; J. Halle, Ottostrasse 3a, Munich, catalogue 33,317 works on mathematics, mostly rare early editions, also 443 works on applied mathematics; Anton Creutzer, Aachen, catalogue 82, mathematics and astronomy; F. L. Dames, Landgrafenstrasse 12, Berlin W., catalogue 81, mathematics and physics; Max Weg, Leplaystrasse 1, Leipsic, catalogue 91, 1581 numbers, including works in applied mathematics and 129 portraits.

Marcus Baker, cartographer of the U. S. Geological Survey and assistant secretary of the Carnegie Institution, died at Washington, December 12. Mr. Baker had been a member of the American Mathematical Society since 1891.

\title{
. NEW PUBLICATIONS.
}

\section{HIGHER MATHEMATICS.}

BALL (R. S.). On the reflection of screw-systems and allied questions. Dublin, 1903. 4to. (Transactions of the Royal Irish Academy, Vol. 32, section A, part 6, pp. 101-154.) 1s. 6d.

BLICHFErdT (H. F.). On the order of linear homogeneous groups. (Transactions of the American Mathematical Society, Vol. 4, pp. 387-397.)

Boole (M. E.). Lectures on the logic of arithmetic. Cambridge, Macmillan and Bowes, 1903. 8vo. Cloth. 2s.

Bowden (J.). Elements of the theory of integers. New York, Macmillan, 1903. 12mo. $10+258 \mathrm{pp}$. Cloth. $\$ 1.25$

BUGAIEv (N. V.). Introduction to analysis and to the differential calculus. Moscow, 1902. 8vo. 204 pp., 1 plate. (Russian.)

Fiedler (W.). See Salmon (G.). 
Fink (E.). Eliah Wilna und sein elementar-geometrisches Compendium. Frankfurt a. M., 1903. 8vo. 29 pp.

Grace (J. H.) and Young (A.). Algebra of invariants. London, Clay, 1903. 8vo. 392 pp. Cloth. 10s.

Grodsky (G. D.). Text-book of analytic geometry. Part I: Plane analytic geometry. St. Petersburg, 1903. 8vo. 210 pp. (Russian.)

M. 4.00

HAGEN (J. G.). Synopsis der höheren Mathematik, Vol. III: Differential- und Integralrechnung. 4te Lieferung. Berlin, Dames, 1903. 4to. Pp. 129-256.

M. 5.00

Hartwig (T.). Transformation sphärischer Punkt- und Linienkoordinaten. (Progr.) Wiener-Neustadt, 1903. 8vo. $22 \mathrm{pp}$.

Heller (T.). Der einfachste Körper, welcher kinetische Geometrie besitzt. (Progr.) Nürnberg, 1903. 8vo. $41 \mathrm{pp}$.

Hochнетм (A.). Aufgaben aus der analytischen Geometrie der Ebene. Heft I: Die gerade Linie, der Punkt, der Kreis. A. Aufgaben. 3te, vermehrte Auflage, bearbeitet von F. Hochheim. Leipzig, Teubner, 1904. 8vo. $6+98$ pp. Cloth. M. 2.40

Houllevigne (T.). Rapport sur le progrès scientifique (mathématiques, physique, chimie), présenté aux assises littéraires et artistiques (3e session, Caen, 4-6 juin 1903). Paris, Valin, 1903. 8vo. $43 \mathrm{pp}$.

Huss (J.). Ueber eine Anwendung der Theorie der linearen Differentialgleichung der Kugelfunktionen einer Variablen. (Diss.) Greifswald, 1903. 8vo. $16 \mathrm{pp}$.

Jessop (C. M.). A treatise on the line complex. London, Clay, 1903. 8vo. 380 pp. Cloth. 10s.

Jones (A. C.). Notes on analytical geometry: an appendix. London, Frowde, 1903. 8vo. $176 \mathrm{pp}$. Cloth. 6s.

KRUG (A.). Die lineare Differentialgleichung dritter Ordnung. (Progr.) Aussig, 1903. 8vo. 60 pp.

LiNNевокN (A.). Die Fokaleigenschaften der Gebilde zweiter Ordnung in der Riemannschen Raumform. Münster, 1902. 8vo. $26 \mathrm{pp}$.

M. 1.80

MerchICH (M.). De veris geometriae integrae principiis contra geometras Euclideos simul et Noneuclideos. Zagrabiae, Merchich, 1903. 8vo. $37 \mathrm{pp}$.

M. 1.00

Miller (G. A.) and Moreno (H. C.). Non-abelian groups in which every subgroup is abelian. (Transactions of the American Mathematical Sociely, Vol. 4, pp. 398-404.) New York, 1903. 8vo.

Moreno (H. C.). See Miller (G. A.).

Rübenstein (N.). Ueber Darstellung von Funktionen durch periodische Reihen. (Progr.) Mähr.-Ostrau, 1903. 8vo. 40 pp.

Salmon (G.). Analytische Geometrie der Kegelschnitte mit besonderer Beriicksichtigung der neueren Methoden. Frei bearbeitet von W. Fiedler. 6te Auflage. Teil 2. Leipzig, Teubner, 1903. 8vo. 24 pp. and pp. 443-854.

M. 8.00 
Seybold (C.). Die Drusenschrift Kitab Alnoqat Waldawair (Das Buch der Punkte und Kreise). Nach dem Münchener und Tübinger Codex. Tübingen, 1902. 4to. $111 \mathrm{pp}$. M. 4.00

SocHock I (I.). Zasady teoryi funkcyj eliptycznych. [Elements of the theory of elliptic functions.] Warszawa, 1903. 8 vo. $50 \mathrm{pp}$.

R. 0.50

Stoffaes. Cours de mathématiques supérieures, à l'usage des candidats à la licence ès sciences physiques. Paris, Gauthier-Villars, 1904. 8vo. $7+538$ pp.

Fr. 10.00

WELD (L. G.). A short course in the theory of determinants. 3d edition. New York, Macmillan, 1903. 12mo. $13+238 \mathrm{pp}$. Cloth.

WoLIETz (K.). Ueber eine von Jakob Steiner aufgestellte birationale geometrische Verwandschaft vom zweiten Grade nebst Anwendung. (Progr.) Jâgernlorf, 1903. 8vo. 39 pp., 1 plate.

Young (A.). See Grace (J. H.).

\section{ELEMENTARY MATHEMATICS.}

Amaldi (U.). See Enriques (F.).

APEL (O.). Ueber die Behandlung einiger mathematischer Kapitel im Unterricht. (Progr.) Halle, 1903. 4tọ. 20 pp. M. 1.20

Augus's (E. F.). Logarithmische und trigonometrische Tafeln. 25ste Auflage, von F. August. Leipzig, 1903. 12mo. $8+204$ pp. Boards.

M. 1.60

Baudoin (P.). Leçons d'arithmétique et d'algèbre, a l'usage des classes de quatrième $\mathrm{B}$ et de troisième $\mathrm{A}$ des lycées et colleges, et de tous les établissements d'instruction. Paris, André, 1903. 8vo. $232 \mathrm{pp}$.

Borel (E.). Algèbre (second cycle). Paris, Colin, 1903. 16mo. $7+379 \mathrm{pp}$.

Fr. 3.00

C. (F. I.). Eléments d' algèbre, avec de nombreux exercises. Paris, Poussielgue, 1903. 16mo. $8+413 \mathrm{pp}$.

Ceramicola (F.). Saggio di geometria intuitiva ad uso dei ginnasi inferiori. Recanati, 1903. 8 vo. 77 pp., 8 plates.

CoRTázar (D. J.). Tratado de trigonometría y topografía. 22a edición, corregida y arreglada por D. J. de Cortázar. Madrid, Perlado, 1904. 8vo. $216 \mathrm{pp}$. Fr. 4.50

DUPORT (J. B.). Lehrbuch der Aritlımetik für die II. und III. Klasse der Mädchen-Lyzeen. Wien, Deuticke, 1903. 8vo. $3+175$ pp. Cloth.

M. 2.25

Lehrbuch der Arithmetik und Geometrie für die 6te Klasse der Mädchen-Lyzeen. Wien, Deuticke, 1903. 8vo. $4+217$ pp. Cloth.

M. 3.00

Dupuis (J.). Tables de logarithmes a sept décimales. Edition stérétype, contenant les logarithmes des nombres de 1 a 100,000, les logarithmes des sinus et des tangentes des angles calcules de seconde en seconde pour les cinq premiers degrés et de dix secondes en dix secondes pour tous les degrés du quart de cercle, et quelques 
tables usuelles. 12e tirage. Paris, Hachette, 1903. 8vo. $12+$ $580 \mathrm{pp}$.

Fr. 3.50

Enriques (F.) e Amalni (U). Elementi di geometria elementare ad uso dei ginnasi superiori. Bologna, Zanichelli, 1903. 16mo. 136 pp.

Fabinger (F.). Základy geometrie Euklidovy. (Progr.) Smichov, 1903. 8vo. $28 \mathrm{pp}$.

Gilbert (J. H.) and SullivaN (E.). Practical lessons in algebra; elementary. Revised edition. New York, Richardson, 1903. $12 \mathrm{mo}$ $6+205+32$ pp. Cloth. $\$ \$ 1.00$

Gómez Pallete (D. J.). Apuntes para el estudio de la trigonometría; descripción detallada de las tablas de Schrön, por L. Iribarren y Arce. 4a edición. Toledo, Gómez Menor, 1903. 8vo. 85 pp., 1 plate.

Fr. 4.00

GraF (H. G.). Wie lehrt und lernt man die Raumlehre? Ein Handbuch für Lehrer und Schüler. Heft 1. Neuwied, Heuser, 1904. 8 vo. $8+60$ pp.

M. 1.20

Hall (H. S.). Short introduction to graphical algebra. 2d edition, revised. Cambridge, Macmillan and Bowes, 1903. 12mo. Cloth.

1s.

Hеснт (B.). Zusammenstellung des mathematischen Lehrstoffes für die untere und mittlere Stufe des Realgymnasiums. Königsberg, 1903. 8vo. $52 \mathrm{pp}$.

M. 1.60

IBRÜGGER (C.). Ableitung einiger Eigenschaften der Kegelschnitte im Anschluss an die bei der Dreieckberechnung vorkommenden Formeln. (Festschrift.) Greifenberg i. P., 1903. 8vo. 14 pp., 1 plate.

M. 1.60

Iribarren y Arce (L.). See Gómez Pallete (D. J.).

KnotT (C. G.) and Mackay (J. S.). Practical mathematics. New edition, revised under supervision. London, Chambers, 1903. 8vo. $640 \mathrm{pp}$. Cloth.

4s. 6 d.

KraUs (K.). Grundriss der geometrischen Formenlehre für LehrerinnenBildungsanstalten. Wien, Pichler, 1903. 8vo. 208 pp. Cloth.

Mackay (J. S.). See KnotT (C. G.).

Martini Zucchani (•A.). Algebra complementare. Livorno, Giusti, 1903. 16mo. $8+143 \mathrm{pp}$. (Biblioteca degli studenti; riassunti per tutte le materie d'esame nei licei, ginnasi, istituti tecnici ecc., Vol. 33-34.) $\quad$ Fr. 1.00

Martus (H. C. E.). Mathematische Aufgaben zum Gebrauche in den oberen Klassen höherer Lehranstalten. (In 4 Teilen.) Teil II: Ergebnisse der Aufgaben in Teil I. 11te Auflage. Dresden, 1903. 8vo. $280 \mathrm{pp}$. M. 4.80

MÉrax (C.). Nouveaux éléments de géométrie. Nouvelle édition, refondue et augmentée. Dijon, Jobard, 1903. 8vo. $8+450$ pp. 22 plates.

Moneno Rey (S.). Principios de aritmética y de geometría. 3a edición. Madrid, Fuentenebro, 1903. 8vo. $102+78$ pp. $\quad$ Fr. 3.50 
Mur (J.). Rešení trojuhelníka kosouhlého polomêrem kruznicê opsané. (Progr.) Rychnov p. Kn., 1903. 8vo. 16 pp.

SAMPERE Y Carreras (J.). Flementos de algebra, conteniendo el cálculo de probabilidades y tablas logarítmicas. Valladolid, Santarén Madrazo, 1903. 8vo. $296 \mathrm{pp}$.

Fr. 7.00

Shmulevich (P. K.). Collection of examination problems in higher schools. Part I: Trigonometry. 4th edition. St. Petersburg, 1903. 8vo. 214 pp., I plate. (Russian.)

SuÁrez Somonte (I.). Nociones de geometría. Madrid, Méndez, 1903. 8vo. $244 \mathrm{pp}$.

Fr. 11.00

Sullivan (E.). See Gilbert (J. H.).

\section{APPLIED MATHEMATICS.}

BASHFORTH (F.). Historical sketch of the experimental determination of the resistance of the air to the motion of projectiles. Cambridge, 1903. 8 vo. 30 pp., 1 plate.

BIENECK (J.). Ueber die astigmatische Abbildung einer unbegrenzten Ebene in einer Kugel bezüglich eines festen Augenpunktes. (Diss.) Rostock, 1903. 8vo. 31 pp., 2 plates.

BRUNNer (E.). Reaktionsgeschwindigkeit in heterogenen Systemen. (Diss.) Göttingen, Vandenhoeck \& Ruprecht, 1903. 8 vo. 66 pp.

M. 1.80

Caldarera (F.). Corso di meccanica razionale. Vol. II: Statica; dinamica. Palermo, Tipografia matematica, 1901. 8vo. $4+$ $357 \mathrm{pp}$.

Coym (A.). Ueber elastische Schwingungen, die durch Austrahlung von Energie gedämpft werden. (Diss.) Berlin, 1903. 8vo. $58 \mathrm{pp}$.

Dumetz (E.). Etudes théoriques et pratiques sur les ponts métalliques à une travée et à poutres droites et pleines. (Ânnexe.) Paris, Beranger, 1903. 8vo. $6+48 \mathrm{pp}$.

ErCHLER (M.). Beiträge zur Theorie der astigmatischen Abbildungen von Objekten in hyperbolischen Spiegeln. Mit Anwendung auf die Abbildung des Sternhimmels. (Diss.) Rostock, 1903. 8vo. 52 pp. 6 plates.

Estanave (E.). Nomenclature des mémoires de physique expérimentale et de physique mathématique présentés en France dans le courant du XIXe siecle, devant les facultés des sciences, en vue du doctorat. Paris, Gauthier-Villars, 1903. 8vo. 20 pp.

FöpPL (A.). Vorlesungen über technische Mechanik. Vol. 2: Graphische Statik. 2te Auflage. Leipzig, Teubner, 1903. 8vo. $12+$ $471 \mathrm{pp}$. Cloth.

M. 10.00

Frischauf (J.). Grundriss der theoretischen Astronomie und der Geschichte der Planetentheorien. 2te, vermehrte Auflage. Leipzig, Engelmann, 1903. 8vo. $15+199$ pp. M. 5.00

Hawkins (C. C.) and Wallis (F.). Dynamo: its theory, design, and manufacture. $3 \mathrm{~d}$ edition, revised and enlarged. London and New York, Macmillan, 1903. 12mo. $13+925$ pp. Cloth. $\$ 3.00$ 
ITES (P.). Ueber die Abhängigkeit der Absorption des Lichtes von der Farbe in krystallisierten Körpern. Gekrönte Preisschrift. (Diss.) Göttingen, Vandenhoeck \& Ruprecht, 1903. 8vo. 82 pp. $\quad$ M. 2.00

JAmieson (A.). Text-book of applied mechanics and mechanical engineering. Vol. 2. 3d edition. Examination questions. London, Griffin, 1903. 8vo. $632 \mathrm{pp.}$ Cloth. 8s. 6d.

KoLl (O.). Geodätische Rechnungen mittels der Rechenmaschine. Halle, Strien, 1903. 8vo. $4+81$ pp. Cloth.

Marchand (L.). Cours de mécanique générale. Louvain, 1903.8 vo. Fr. 5.00

MATthiessen (L.). Die astigmatische Brechung der Sonnenstrahlen im Regenbogen, mit Anwendung der Kettenbruch-Determinanten dargestellt. Rostock, $1903 . \quad 4$ to. 21 pp., 5 plates.

Murani (O.). Onde hertziane e telegrafo senza fili. Milano, Hoepli, 1903. 16mo. $15+341$ pp. (Manuali Hoepli.) Fr. 3.50

Nerlson (R. M.). The steam turbine. 2d edition, revised and enlarged. New York, Longmans, 1903. 8vo. $17+294$ pp. Cloth.

$\$ 3.60$

Saussure (R. DE). Hypothèse sur la constitution géométrique de l'ether. (Archives des Sciences Physiques et Naturelles, $4 \mathrm{e}$ période, Vol. 16, pp. 369-387.) Paris, 1903.

SchlitT (R.). Untersuchungen über einen Specialfall des Problems der drei Körper mit nahezu periodischer Lösung. (Diss.) Kiel, 1903. 8vo. $30 \mathrm{pp}$. 3 plates.

Steinmetz (C. P.). Theorie et calcul des phénomènes du courant alternatif. Traduit sur la troisième édition américaine, revue et augmentée. Paris, Dunod, 1903. 8vo. $20+526$ pp. Fr. 20.00

Tetmajer (L, voN). Die angewandte Elastizitäts- und Festigkeitslehre, auf Grund der Erfahrung bearbeitet. 2te, vollständig umgearbeitete Auflage. Wien, Deuticke, 1904. 8vo. $15+565$ pp., 10 plates.

M. 16.00

Vollgrafe (J. A.). Over de theorie der electromagnetische draaingen en der unipolaire inductie. Leiden, 1903. 8vo. 178 pp. M. 6.00

WALKer (T.). Introduction to physical chemistry. 3d edition. New York, Macmillan, 1903. 8vo. $12+368$ pp. Cloth. $\$ 3.25$

Wallis (F.). See Hawkins (C. C.).

WERNICKe (AD.). Lehrbuch der Mechanik in elementarer Darstellung mit Anwendungen und Uebungen aus den Gebieten der Physik und Technik. Teil I. 3te (Schluss-) Abteilung. 4te, völlig umgearbeitete Auflage, von Al. Wernicke. Braunschweig, Vieweg, 1903. 8vo. $11 \mathrm{pp}$. and pp. 811-1635.

WITz (A.). Traité théorique et pratique des moteurs à gaz et $\mathfrak{a}$ petrole. 4e edition. 2 volumes. Paris, 1903. 8vo. 500 pp. 